\title{
Poly(3-alkylthiophenes): new sorption materials for solid phase microextraction of drugs isolated from human plasma
}

\author{
Pawel Olszowy • Malgorzata Szultka • \\ Boguslaw Buszewski
}

Received: 23 March 2011 /Revised: 30 May 2011 / Accepted: 2 June 2011 /Published online: 28 June 2011

(C) The Author(s) 2011. This article is published with open access at Springerlink.com

\begin{abstract}
A novel sorbent in solid phase microextraction (SPME) method based on poly(3-alkylthiophenes) was used in the isolation of linezolid from human plasma samples following liquid chromatography determination. The effect of extraction time on the sorption capacity of the SPME process was studied and pointed at $10 \mathrm{~min}$ both for adsorption and desorption. Poly(3-methylthiophene) and poly(3-nonylthiophene) were applied for the extraction of linezolid from water solutions. In plasma samples, four coatings including polythiophene and poly(3-penthylthiophene) were investigated. With these measurements, correlation coefficients were calculated in the range from 0.9820 to 0.9995 , and the relative standard deviations were below $15 \%$. That allowed claiming that the synthesized and described materials can be successfully applied in the analysis of linezolid also from other matrices such as urine or blood.
\end{abstract}

Keywords Solid phase microextraction · Poly(3alkylthiophenes) - Linezolid · High-performance liquid chromatography

\section{Introduction}

Solid phase microextraction (SPME) belongs to one of the fastest developing methods in analytical chemistry. A solventless technique invented by Arthur and Pawliszyn

P. Olszowy $\cdot$ M. Szultka $\cdot$ B. Buszewski $(\square)$

Department of Environmental Chemistry and Bioanalytics,

Faculty of Chemistry, Nicolaus Copernicus University,

Gagarin 7 Street,

87-100 Torun, Poland

e-mail: bbusz@chem.umk.pl
$[1,2]$ allows for visible speed-up sample preparation process and direct quantitations/quantification of analytes with the use of the GC or LC system. At the beginning, the sorbents most often used were based on polyacrylates and polysiloxanes coated on the support with use of deep coating [3]. Hence, solid phase microextraction was used at the beginning for the analysis of low-molecular-weight analytes from environmental matrices and biological matrices in subsequent studies as well $[4,5]$. Because of the increasing demand of sorption materials used in SPME, developing a new kind of material is still necessary $[3,6]$.

Polymers containing conjugated bond systems have played an important role in the sample preparation method and sample analysis for more than decade. Up to this time, two of them (polypyrrole and polythiophene) have found wide application in SPME [7-10] as sorbent materials. A simple and fast approach allowing to prepare polymeric sorbents based on this kind of polymers is electropolymerization. This method allows obtaining fibers which may be further applied in the solid phase microextraction of biologically active compounds from various biological matrices. Such coatings may be applied in online desorbing units for high-performance liquid chromatography (HPLC) applications as well as in injection devices for GC.

In the present work, a stainless steel SPME fiber coated with different poly(3-alkylthiophenes) was made through an electrochemical polymerization for the first time. The fibers were applied for the extraction of drug from oxazolidinone groups (linezolid) as a model compound from standard solutions and human plasma samples. Antibiotics from this group are widely used in bacterial infection treatment. Coatings, which were used in measurements, were characterized by a different lipophilicity, which has a significant influence on the extraction efficiency, and then selectivity, of the applied drug. The obtained results were used in the 
statistical evaluation to exhibit the most appropriate coatings in the extraction of linezolid and indicate chemical similarities among all used fibers. Application of polymeric SPME coatings characterized by different $\log P$ values also shows changes in selectivity toward the applied model compounds. This will help select the appropriate thiophene alkyl derivatives in the sampling of different compounds in the future. Additionally, the creation of fibers with different hydrophobicities based on the same polymer may show changes in the surface properties and, hence, ability to extract chosen analytes.

\section{Experimental}

\section{Chemicals}

All chemicals and reagents were HPLC or analytical grade. Monomer thiophene (99\%) and 3-methylthiophene (98\%) were purchased from Sigma-Aldrich (Schnelldorf, Germany) and used without further purification. Linezolid $((S)-N-[[3-[3-$ fluoro-4-(4-morpholinyl) phenyl]-2-oxo-5-oxazolidinyl] methyl]-acetamide) and drug-free human plasma were kindly provided by Pharmacia \& Upjohn GmbH (Erlangen, Germany) and Collegium Medicum (Nicolaus Copernicus University, Torun, Poland), respectively.

A $125-\mu \mathrm{g} / \mathrm{mL}$ stock solution of the linezolid was prepared in methanol. Working solutions were prepared by appropriate dilution of the stock solution in distilled water. Methanol for HPLC was obtained from J.T. Baker (Deventer, the Netherlands). Water was obtained by means of a Milli-Q RG apparatus from Millipore (Millipore Intertech, Bedford, MA, USA) in our laboratory.

\section{Apparatus}

The chromatographic system was a HPLC 1100 (Agilent, Waldbronn, Germany). It consisted of a quaternary pump, a degasser, an automatic sample injection, and a variablewavelength UV-Vis detector. An Agilent Triple Quad mass spectrometer with an electrospray ionization (ESI) interface was used. For sample evaporation, a Labconco CentriVap DNA concentrator (Kansas City, KS, USA) was used.

Electropolymerization was performed using a homemade setup system coupled with high-performance potentiostat/galvanostat PGSTAT128N series Autolab model (Utrecht, the Netherlands). The surface studies of poly(3alkylthiophenes) were investigated using a scanning electron microscope (SEM) LEO 1430VP (Carl Zeiss SMT, Oberkochen, Germany).

Theoretical $\log P$ values of poly(3-alkylthiophenes) were determined after geometry optimization by applying HyperChem software (Hypercube, Canada).
Synthesis of monomers and preparation of SPME poly(3-alkylthiophene) coatings

In this work, we synthesized 3-pethylthiophene and 3nonylthiophene, which were next electropolymerized to obtain SPME coatings. Synthesis was performed according to the well-known Grignard reaction, which basically relies on coupling the appropriate Grignard compound (organomagnesium compound) with other bromide derivatives (in our case, 3-bromothiophene). Such coupling leads to obtaining an average efficiency about $50-55 \%$ when an excess of Grignard compound is used. These reactions were made according to the procedure described earlier [11, 12]. Monomers were purified by distillation under low pressure $(5 \mathrm{mmHg})$. The boiling temperature ranges for 3-pentylthiophene and 3-nonylthiophene were 55-58 and $108-112{ }^{\circ} \mathrm{C}$, respectively. The purity of the synthesized compounds was checked using ${ }^{1} \mathrm{H}$ and ${ }^{13} \mathrm{C}$ NMR.

In order to ensure coating reproducibility, all poly(3alkylthiophene) coatings were synthesized using an identical electrochemical cyclic voltammetric procedure in a three-electrode cell. The system was operated at a scan rate $50 \mathrm{mV} / \mathrm{s}$ in a potential range from -0.2 to $+3.0 \mathrm{~V}$. The number of scans was 5 . Polythiophene was obtained using the same procedure, but the potential range was from -0.2 to $2.5 \mathrm{~V}$ and the number of scans was 12 . All polymerizations were performed in $0.25 \mathrm{M}$ tetrabutylammonium tetrafluoroborate, in which monomer concentrations were $0.1 \mathrm{M}$ each time.

Table 1 shows the chemical structure of various polythiophene derivatives including poly(3-methylthiophene), poly(3-penthylthiophene), poly(3-nonylthiophene), and pure polythiophene.

HPLC and UV spectrophotometry/mass spectrometry (UV/MS)

A volume of $15 \mu \mathrm{l}$ of the prepared sample was injected for HPLC analysis. HPLC was carried out using a guarded $(10.0 \times 4.6-\mathrm{mm})$ column of $\mathrm{ACE}^{\circledR}$ ODS $150 \times 4.6 \mathrm{~mm}, 300$ Angstrom $(\AA)$ pore size, $5-\mu \mathrm{m}$ particle diameter, and an isocratic elution at a flow rate of $450 \mu \mathrm{l} / \mathrm{min}$. The mobile phase comprised 50:50 water/methanol. Wavelength was set at $\lambda=251 \mathrm{~nm}$.

Additionally, the chromatographic system was coupled with a mass spectrometer $\left(\mathrm{MS}^{\mathrm{n}}\right)$ equipped with an ESI interface and operated with the Mass Hunter software. Negative ion selected ion monitoring (SIM) mode was used to detect and verify the chemical and molecular structure of linezolid among the other chemicals in human plasma samples. The MS conditions were as follows: ESI in positive mode; capillary temperature, $320{ }^{\circ} \mathrm{C}$; dwell time, $200 \mathrm{~ms}$; nitrogen flow, $8.5 \mathrm{~L} \mathrm{~min}^{-1}$; nebulizer pressure, 
Table 1 Chemical structures and thicknesses of the prepared SPME fibers

\begin{tabular}{|c|c|c|c|}
\hline SPME fibers & Abbreviation & Chemical structure & Thickness $[\mu \mathrm{m}]$ \\
\hline Polythiophene & PTh & & \\
\hline Poly(3-methylthiophene) & PMeTh & & \\
\hline Poly(3-penthylthiophene) & PPentTh & & \\
\hline Poly(3-nonylthiophene) & PNonTh & & \\
\hline
\end{tabular}

30 psi. Acquisition at full scan and SIM was performed at $m / z=338.5$ corresponding to $[\mathrm{M}+\mathrm{H}]^{+}$.

\section{SPME of linezolid from human plasma}

SPME was carried out with the synthesized poly(3alkylthiophene) fibers. Before the extraction, the SPME fibers were conditioned for $10 \mathrm{~min}$. Extractions were performed by exposing a $1.5-\mathrm{cm}$ length of the relevant SPME coating into the $1.5-\mathrm{mL}$ sample solution. To optimize the extraction condition, extraction time ranging from 1 to 20 min was studied. Desorption was carried out by inserting the SPME fiber into the pure $\mathrm{MeOH}$ for $10 \mathrm{~min}$.

\section{Calibration and validation of the assay}

Calibrations were calculated by linear regression of the signal area of linezolid vs. the applied corresponding concentrations. The calibrators contained linezolid at final concentrations from 3 or $1 \mu \mathrm{g} \mathrm{mL} \mathrm{m}^{-1}$ for plasma up to $20 \mu \mathrm{g} \mathrm{mL}^{-1}$. Each calibration function was based on six calibrators prepared and measured in triplicate at least. The method validation procedures were carried out according to the Food and Drug Administration (FDA) guidelines [13].
The assays were repeated three times within the same day to obtain repeatability (intraday precision), expressed as a percentage relative standard deviation values (RSD). The limit of detection ( $\mathrm{LOD}=3 \times \mathrm{SD} x y / b$, where $\mathrm{SD} x y$ is the standard deviation and $b$ is the slope) and the limit of quantification $(\mathrm{LOQ}=10 \times \mathrm{SD} x y / b)$ were calculated with acceptable precision and accuracy.

\section{Results and discussion}

Preparation and characterization of extracting phases

Depending on the nature of the side chains, various functionalized polythiophenes can be obtained, but in the present study, poly(3-methylthiopene), poly(3-penthylthiophene), and poly(3-nonylthiophene) were applied. Poly(3alkylthiophene) fibers were obtained by electrochemical polymerization using the described three-electrode cell. For each of them, anodic oxidation of the electroactive monomers was carried out. The conditions of this process were optimized separately for preparing polythiophene derivative materials.

The selected materials are characterized by a diverse lipophilicity according to the different chain alkyl lengths in 

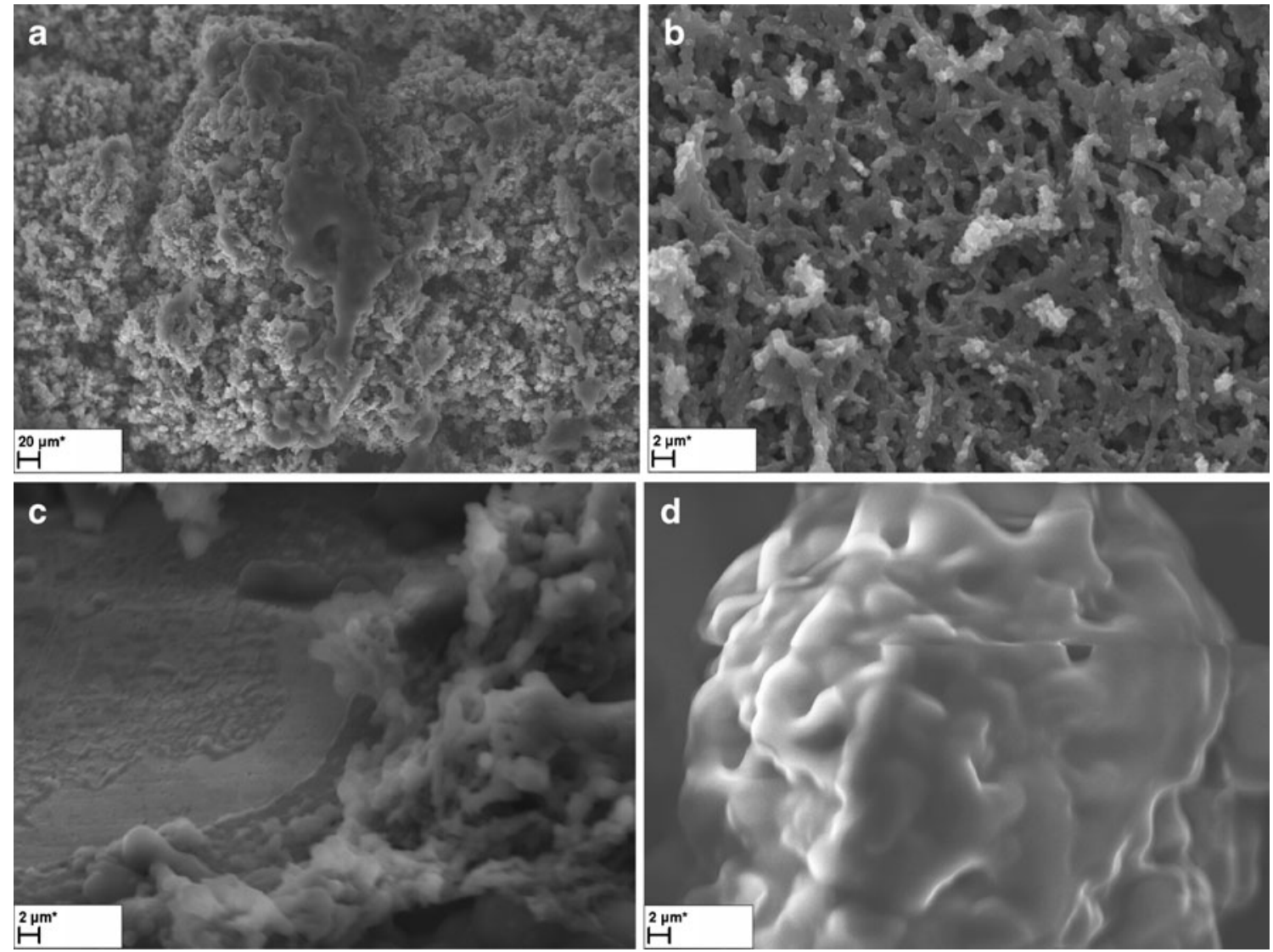

Fig. 1 Scanning electron micrograph of polythiophene (a), poly(3-methylthiophene) (b), poly(3-penthylthiophene) (c), and poly(3nonylthiophne) (d)

their structure. $\log P$ was in range from 1.90 for pure thiophene to 6.61, which was calculated for 3-nonylthiophene. A good correlation between the hydrophobicity possessed by applying SPME coatings and the number of the carbon atoms in their side alkyl chains has been obtained $\left(R^{2}=0.9998\right)$.

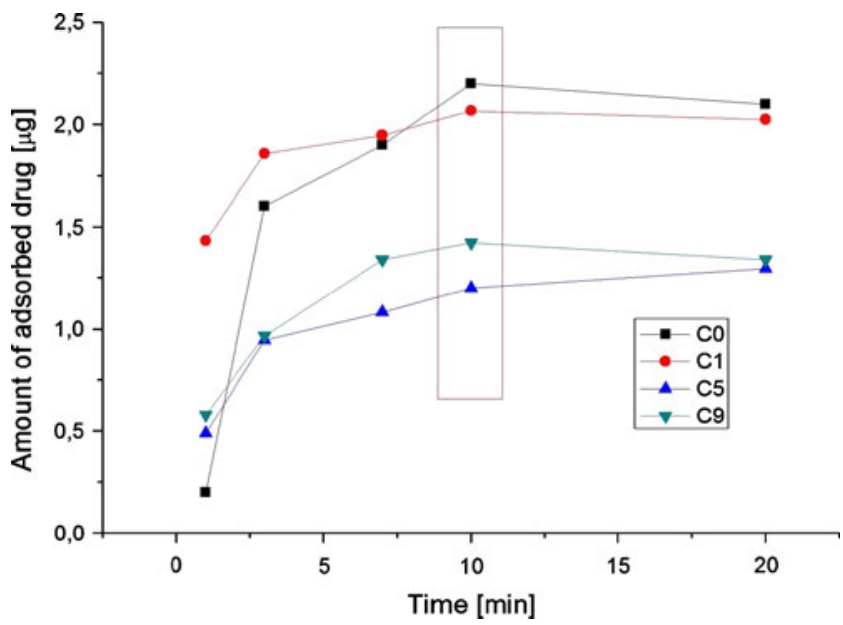

Fig. 2 Adsorption time profile of linezolid from aqueous solutions
Electrochemical polymerization of thiophene and three alkyl derivatives made it possible to compare the oxidation potentials of these materials. Based on the voltamperograms, it is possible to discern a specific trend in the oxidation potential of the relevant monomer with increasing

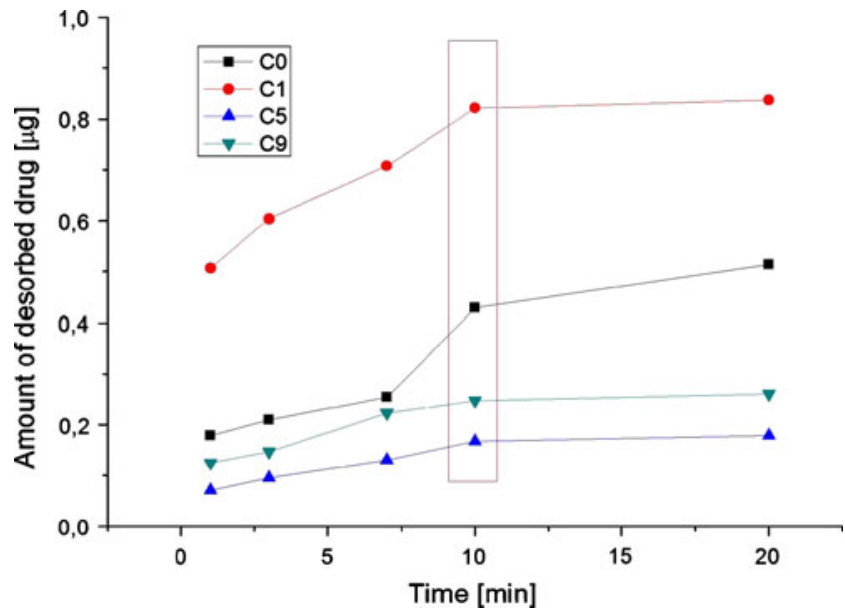

Fig. 3 Desorption time profile of linezolid from aqueous solutions 


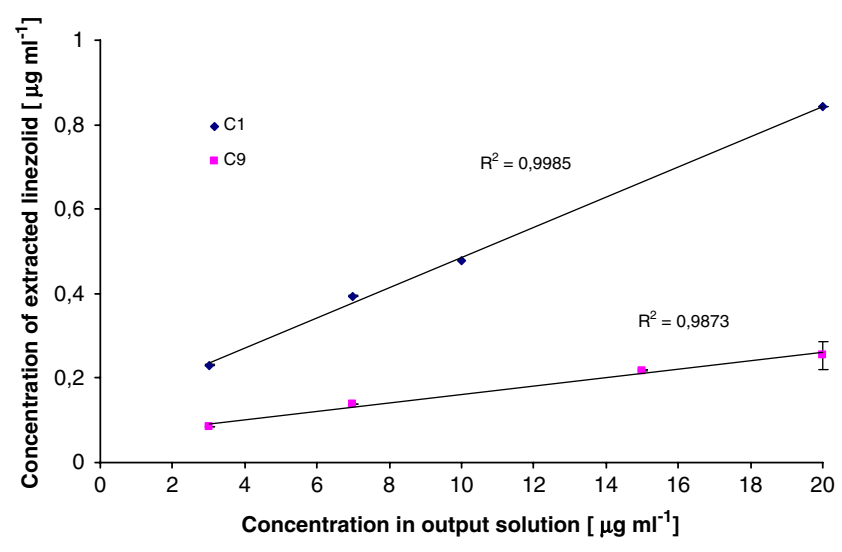

Fig. 4 Dependence amount of extracted linezolid vs. concentration in output aqueous solutions

alkyl chain length. The necessary potential to initiate the oxidation of the monomer was in range between $0.75 \mathrm{~V}$ for polythiophene and $1.25 \mathrm{~V}$ in the case of poly(3-nonylthiophene). This clearly shows that the length of alkyl chain in 3-alkylthiophene possesses a directly proportional effect on the oxidation potential of the applied monomer. The determination coefficient of this dependence is 0.9997 .

Investigations made using of SEM allow measuring fiber thickness. Additionally, in the case of poly(3methylthiophene), images showed differences in mor- phology and porosity. The structure of poly(3-methylthiophene) fibers presented in Fig. 1 allow seeing some similarities with polymeric monolith structure widely described in the paper about liquid chromatography [14]. In accordance with increasing alkyl chain length, a significant decrease of structure order was observed (Fig. 1b). Such huge morphology, estimated based on SEM images, obviously decreased with growing of the alkyl side chains. Therefore large changes in the surface properties of fibers will for sure have an influence on the sorption properties exhibited in relation to the antibiotic chosen for further investigations.

In the present study, hydrophobic-hydrophilic interactions are the primary driving force for the extraction of linezolid. As the introduction of the functional groups onto conductive polymers can affect different selectivities and behaviors of the extraction phase toward the target compound, the use of poly(3-alkylthiophene) derivatives seems to be reasonable for the uptake of hydrophilic compounds.

Optimization of the poly(3-alkylthiophene) extraction time

SPME variables, such as adsorption and desorption time, were optimized to perform the target analyte sorption equilibrium during the optimal period of time. The
Fig. 5 Three-dimensional graph plot presenting the SPME coatings as a function of changes made in the type of applied concentration of linezolid or the amount of extracted drug from plasma samples

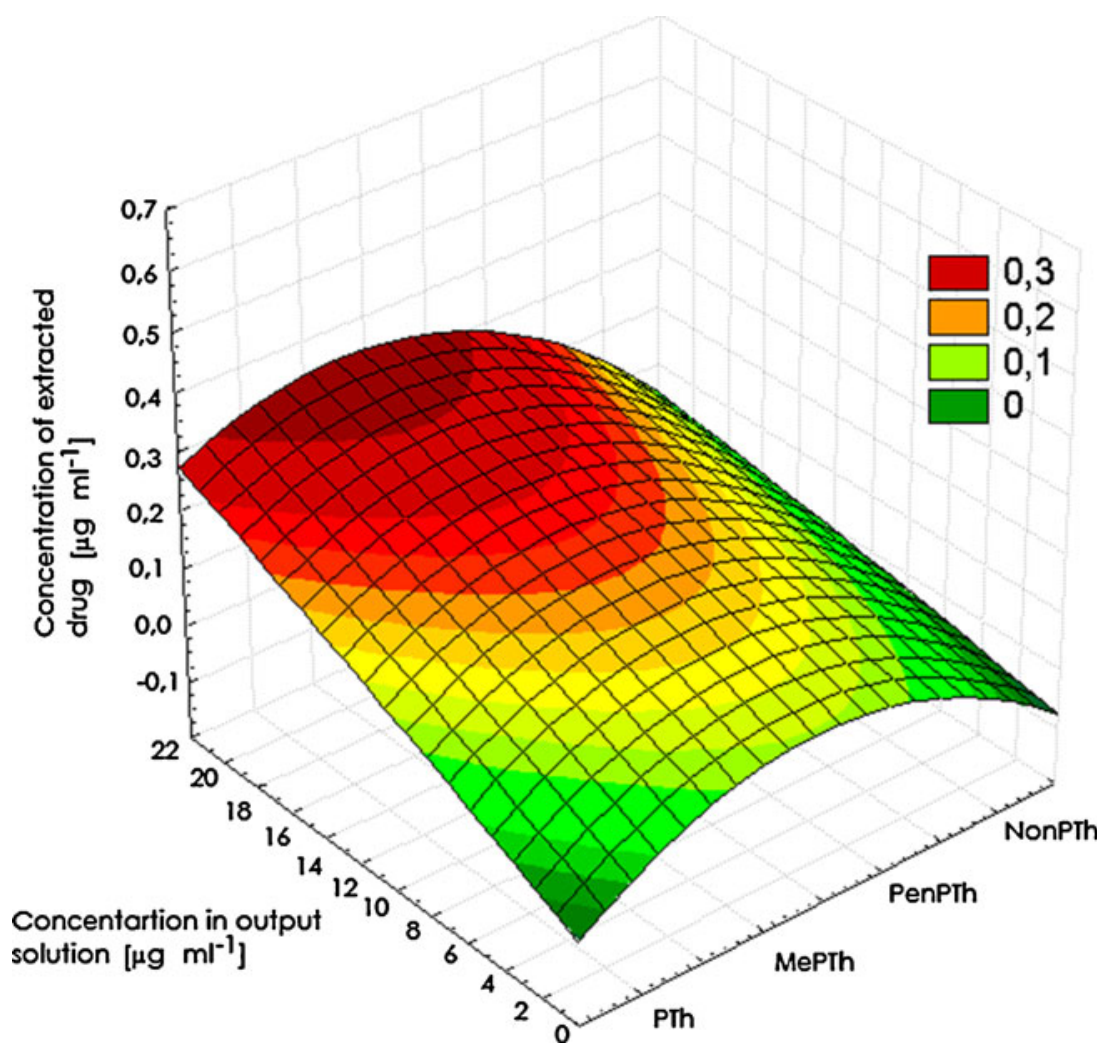


Table 2 Comparison of the amount of extracted drug by different SPME coatings in human plasma samples $(n=3)$

\begin{tabular}{lllll}
\hline Concentration $\left(\mu \mathrm{g} \mathrm{mL}^{-1}\right)$ & \multicolumn{4}{l}{ Concentration of extracted linezolid $(\mu \mathrm{g})$} \\
\cline { 2 - 5 } & PTh & PMeTh & PPentTh & PNonTh \\
\hline 1 & 0.081 & 0.055 & 0.043 & 0.054 \\
3 & 0.089 & 0.129 & 0.056 & 0.066 \\
7 & 0.094 & 0.159 & 0.061 & 0.099 \\
10 & 0.121 & 0.401 & 0.075 & 0.097 \\
15 & 0.139 & 0.465 & 0.094 & 0.128 \\
20 & 0.154 & 0.598 & 0.119 & 0.145 \\
$\mathrm{SD}\left(\mu \mathrm{g} \mathrm{mL}^{-1}\right)$ & $0.0007-0.0036$ & $0.0009-0.0228$ & $0.0001-0.00134$ & $0.0003-0.0319$ \\
$R$ & 0.9971 & 0.9996 & 0.9812 & 0.9974 \\
\hline
\end{tabular}

extraction time is an important SPME parameter in achieving equilibrium in the distribution of analytes between the fiber and sample. For this purpose, each SPME fiber was exposed to an aqueous solution of linezolid (15 $\mu \mathrm{g} \mathrm{mL}^{-1}$ ) for a different time from 1 to $20 \mathrm{~min}$. Variations of the amounts of extracted drug as a function of the extraction time (adsorption/desorption) are presented in Figs. 2 and 3.

From Figs. 2 and 3, it is clearly seen that the highest adsorbed and desorbed amount of linezolid was obtained at $10 \mathrm{~min}$ of adsorption and desorption. This extraction time was used in all further measurements.

Application of the SPME method in the analysis of linezolid from aqueous solutions and plasma samples

\section{Aqueous solutions}

Experiments performed in aqueous solutions allow getting a dependence, which indicates the sorption capacity of

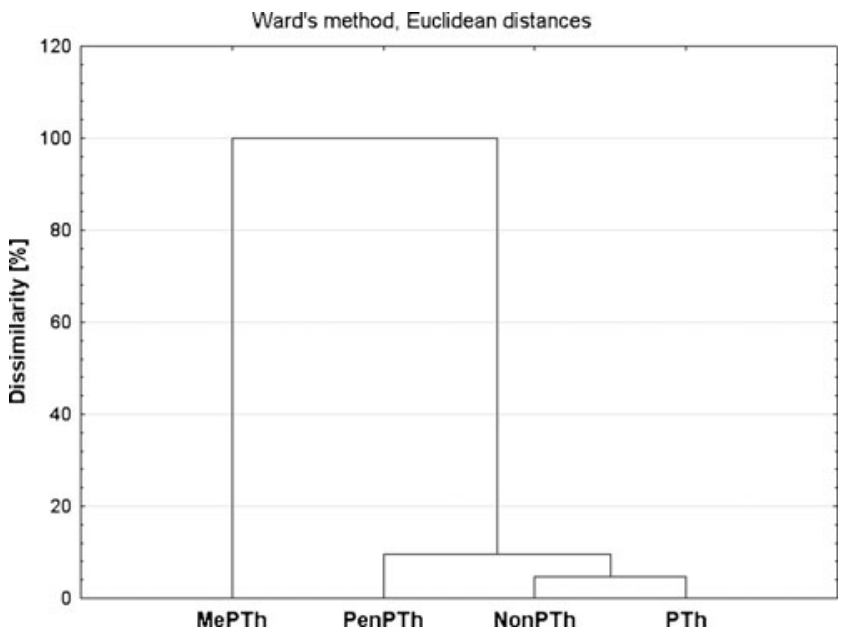

Fig. 6 Dissimilarity dendrogram of the studied SPME coatings related to the amount of extracted linezolid from human plasma samples linezolid to the applied poly(3-alkylthiophene) SPME coatings. The results presented in Fig. 4 reveal the highest extraction abilities of the target compound on the poly(3methylthiophene) surface. The amounts of the extracted drug at the lowest $\left(3 \mu \mathrm{g} \mathrm{mL} \mathrm{m}^{-1}\right)$ and the highest $\left(20 \mu \mathrm{g} \mathrm{mL} \mathrm{mL}^{-1}\right)$ concentrations range from 0.231 to $0.844 \mu \mathrm{g}$, respectively. Correlation coefficients $\left(R^{2}\right)$ for target compound range from 0.9873 for poly(3-nonylthiophene) to 0.9985 in the case of poly(3-methylthiophene) coatings. The RSDs calculated for investigations in aqueous solutions which were in range $0.3-1.9 \%$ showed that repeatability of adsorption with both used SPME fibers was very high.

\section{Human plasma samples}

Extractions of linezolid from human plasma samples were performed using all four sorbent materials. A plot presented in Fig. 5 shows changes of the extracted amount of linezolid depending on the concentration of linezolid in plasma samples. From this plot, it may be clearly seen that the highest extraction efficiency was obtained while using poly(3-methylthiophene) fibers. From this plot, some similarities in the amount of extracted linezolid between polythiophene and poly(3-nonylthiophene) are observed. The amounts of extracted drug with the use of these fibers were on the lowest level.

In Table 3, all results were obtained with the extraction in plasma samples. From these results, it may be concluded that the structure and physicochemical properties of poly(3methylthiophene) possessed the most optimal parameters for the extraction of linezolid. This fact was also confirmed by a correlation coefficient which, in the case of these materials, was 0.9996 .

The results obtained of the extraction in plasma samples presented in Table 2 were utilized for statistical evaluation. Ward's method for data agglomeration and Euclidean distances for similarity measurements have been applied in the cluster analysis as well. The results presented in 
Table 3 Linearity of the SPME/ HPLC method

\begin{tabular}{lllll}
\hline Kind of coating & $\begin{array}{l}\text { Linear regression } \\
x, y\left(\mu \mathrm{mL}^{-1}\right)\end{array}$ & $R$ & $\begin{array}{l}\text { Linear range } \\
\left(\mu \mathrm{gL}^{-1}\right)\end{array}$ & RSD \\
\hline Polythiophene & $y=0.0039 x+0.0782$ & 0.9971 & $1-20$ & $0.3-5.7$ \\
Poly(3-methylthiophene) & $y=0.0283 x+0.0357$ & 0.9996 & \\
Poly(3-penthylthiophene) & $y=0.0035 x+0.0452$ & 0.9812 & \\
Poly(3-nonylthiophene) & $y=0.0048 x+0.0513$ & 0.9974 & \\
\hline
\end{tabular}

Fig. 6 show that all sorbents may be divided into two groups exhibiting different extraction properties and different selectivities toward the linezolid. Poly(3-methylthiophene), which was mentioned earlier, allows obtaining the highest extraction efficiencies and shows the highest selectivity toward the applied drug. The other three materials were characterized to exhibit lower extraction efficiencies. The amounts of extracted linezolid according to increasing concentrations of linezolid in output solutions were also very similar.

\section{Analytical validation of the developed method}

Calibration curves were set up for target coatings. A good linearity was found for each of them in the whole concentration ranges. Precision was also satisfactory, with RSD values lower than $5.7 \%$ for each of the tested SPME coating. Calibration curve parameters, presented in Table 3, showed good correlation coefficients $(R)$ from 0.9812 to 0.9996. Additionally, differences in slopes presented in Table 3 proved the higher selectivity of poly(3-methylthiophene) fiber in comparison with the other applied coatings.

The parameters that described the concentration of extracted linezolid vs. concentration in output plasma samples were collected and are presented in Table 3. To complete the validation method, accuracy and RSD values for three concentrations of linezolid and applied SPME coatings were also calculated and are shown in Table 4. The values of method accuracy are in agreement with the relevant FDA guidelines which suggested the mean value to be within $15 \%$ of the actual value [13].

\section{Conclusions}

Application of poly(3-alkylthiophene) SPME coatings, followed by liquid chromatography, provided a simple and sensitive tool for the analysis of linezolid in human plasma samples. The performed analysis in water solutions and plasma samples allow obtaining correlation coefficients in the range 0.9936-0.9992 (water solutions) and 0.98120.9996 (plasma samples). Experiments from aqueous solution and plasma samples obtained better extraction efficiencies toward linezolid by using poly(3-methylthiophene) fibers. The other coatings allow obtaining a high correlation coefficient, but the extraction efficiencies using these fibers were much smaller. Selectivity toward linezolid was the highest in the case of poly(3-methylthiophene), which might be caused by the most regular monolithic structure.
Table 4 Performance of developed method at three concentrations of linezolid $(n=3)$

\begin{tabular}{lrcrc}
\hline Kind of coating & $\begin{array}{l}\text { Nominal conc. } \\
\left(\mu \mathrm{g} \mathrm{mL}^{-1}\right)\end{array}$ & $\begin{array}{l}\text { Found conc. } \\
\left(\mu \mathrm{g} \mathrm{m}^{-1}\right)\end{array}$ & Accuracy (\%) & RSD \\
\hline Polythiophene & 1 & $0.92 \pm 0.00$ & 91.89 & 0.9 \\
& 10 & $9.88 \pm 0.03$ & 98.79 & 3.3 \\
Poly(3-methylthiophene) & 20 & $19.85 \pm 0.09$ & 99.23 & 2.6 \\
& 1 & $0.95 \pm 0.00$ & 94.49 & 0.2 \\
Poly(3-penthylthiophene) & 10 & $10.03 \pm 0.02$ & 100.3 & 1.7 \\
& 10 & $19.40 \pm 0.06$ & 97.01 & 2.3 \\
Poly(3-nonylthiophene) & 10 & $0.94 \pm 0.05$ & 98.01 & 0.1 \\
& 10 & $9.92 \pm 0.07$ & 99.24 & 5.7 \\
& 10 & $19.88 \pm 0.11$ & 99.40 & 1.9 \\
& 20 & $0.94 \pm 0.05$ & 94.47 & 2.9 \\
\hline
\end{tabular}


Acknowledgments The authors would like to thank Dr. Jacek Nowaczyk for helpful and inspiring discussions with synthesis of the applied monomers and Ms. Agnieszka Reczuch for language corrections. This work was supported by European Social Found, Polish National Budget, Kujawsko-Pomorskie Voivodship Budget (within Sectoral Operational Programme Human Resources) "Krok w przyszlosc".

Open Access This article is distributed under the terms of the Creative Commons Attribution Noncommercial License which permits any noncommercial use, distribution, and reproduction in any medium, provided the original author(s) and source are credited.

\section{References}

1. Arthur CL, Pawliszyn J (1990) Solid phase microextraction with thermal desorption using fused silica optical fibers. Anal Chem 62:2145-2148

2. Pawliszyn J (1997) Solid-phase microextraction: theory and practice. Wiley-VCH, New York

3. Kataoka H (2005) Recent advances in solid-phase microextraction and related techniques for pharmaceutical and biomedical analysis. Curr Pharm Anal 1:65-84

4. Ligor M, Buszewski B (1999) Determination of menthol and menthone in food and pharmaceutical products by solid-phase microextraction-gas chromatography. J Chromatogr A 847:161-169

5. Gorog S (2007) The changing face of pharmaceutical analysis. Trends Anal Chem 26(1):12-17
6. Lord H, Pawliszyn J (2000) Evolution of solid-phase microextraction technology. J Chromatogr A 885:153-193

7. Pawliszyn J (1995) New directions in sample preparation for analysis of organic compounds. Trends Anal Chem 14(3):113122

8. Olszowy P, Szultka M, Ligor T, Nowaczyk J, Buszewski B (2010) Fibers with polypyrrole and polythiophene phases for isolation and determination of adrenolytic drugs from human plasma by SPME-HPLC. J Chromatogr B 878:2226-2234

9. Olszowy P, Szultka M, Fuchs P, Kegler R, Mundkowski R, Miekisch W, Schubert J, Buszewski B (2010) New coated SPME fibers for fast HPLC determination of medically important drugs from human blood. J Pharm Biomed Anal 53:1022-1027

10. Buszewski B, Olszowy P, Ligor T, Szultka M, Nowaczyk J, Jaworski M, Jackowski M (2010) Determination of adrenolytic drugs by SPME-LC-MS. Anal Bioanal Chem 397:173-179

11. Czerwinski W, Kreja L, Chrzaszcz M, Kazubski A (1994) Structural and electrical properties of soluble conducting poly(3pentyl-thiophene). J Mater Sci 29(5):1191-1197

12. Czerwinski W, Nowaczyk J, Kania K (2003) Ozonization of electronic conducting polymers. I. Copolymers based on poly[3nonylthiophene]. Polym Degrad Stab 80(1):93-101

13. FDA Guidance for Industry (2001) Analytical procedures and methods validation

14. Courtois J, Szumski M, Bystrom E, Iwasiewicz A, Shchukarev A, Irgum K (2006) A study of surface modification and anchoring techniques used in the preparation of monolithic microcolumns in fused silica capillaries. J Sep Sci 26:14-24 